\section{The systemic absorption of adrenaline from posterior segment infusion during vitreoretinal surgery}

P. HEYWORTH, R. BOURKE, C. MOORE,

N. MORLET, R. COOLING

\begin{abstract}
Background/purpose Adrenaline infused into the posterior segment of the eye during vitreoretinal surgery may be absorbed and give rise to unwanted cardiovascular effects. We sought to establish whether significant amounts of adrenaline are systemically absorbed.
\end{abstract}

Methods This study was prospective and double-masked, with patients randomised into two groups. The first group received a posterior segment infusion containing Hartmann's solution alone (Hs group) and the second group received a posterior segment infusion of Hartmann's solution containing $0.5 \mathrm{ml}$ of 1:1000 $(0.5 \mathrm{mg})$ adrenaline per $500 \mathrm{ml}$ (Hs+Ad group). Pre-medication and anaesthetic techniques were standardised for all patients. Venous blood samples were collected prior to induction, 5 min following intubation and 5, 10, 15 and $30 \mathrm{~min}$ following the commencement of the infusion. Samples were analysed for adrenaline levels using high-performance liquid chromatography.

Results Ten patients were studied: 4 in the Hs group and 6 in the Hs+Ad group. The mean dose of adrenaline administered in the $\mathrm{Hs}+\mathrm{Ad}$ group was $655.08 \mathrm{nmol}(0.12 \mathrm{mg})$. The median serum adrenaline level following infusion for the $\mathrm{Hs}+\mathrm{Ad}$ group was $0.15 \mathrm{nmol} / \mathrm{l}(\mathrm{LQ}=\mathbf{0 . 1 0 0}$, $\mathrm{UQ}=0.360$ ) and the median serum adrenaline level for the $\mathrm{Hs}$ group was $0.10 \mathrm{nmol} / \mathrm{l}$ $(L Q=0.100, U Q=0.350)$. There was no overall statistical difference in the levels of serum adrenaline between the two groups, and furthermore adrenaline levels remained within physiological parameters for both groups.

Conclusion There does not appear to be significant adrenaline absorption from posterior segment infusion. Continued caution should be exercised, however, as idiosyncratic reactions may still occur.

Key words Absorption, Adrenaline, Posterior segment infusion, Vitreoretinal surgery
Patients undergoing pars plana vitrectomy receive a posterior segment infusion of Hartmann's solution containing added adrenaline to maintain mydriasis and assist haemostasis. Since topical adrenaline has been found to be absorbed systemically, ${ }^{1-4}$ it might be expected that adrenaline infused into the posterior segment may similarly be absorbed. As well as the routes by which topical adrenaline may be absorbed there is the potential for further absorption occurring via the ciliary body, iris and choroidal vasculature.

We aimed to find out whether there was significant absorption of administered adrenaline.

\section{Methods}

The study followed the tenets of the Helsinki Declaration. Patients receiving topical or systemic beta-blockers, systemic alpha-blockers, monoamine oxidase inhibitors and halothane anaesthesia or those with significant cardiac dysrhythmias or systemic hypertension were excluded from the study. Patients were randomised into two groups. The first group $(\mathrm{Hs}+\mathrm{Ad})$ received a posterior segment infusion of Hartmann's solution containing $0.5 \mathrm{ml}$ of 1:1000 strength adrenaline $(0.5 \mathrm{mg})$ per $500 \mathrm{ml}$. The second group (Hs) received Hartmann's solution alone.

Patients received pre-medication of temazepam with dosage adjusted (10 $\mathrm{mg}$ per $35 \mathrm{~kg}$ ) to body weight. General anaesthesia was standardised in an attempt to stabilise endogenous adrenaline secretion. Prior to induction of anaesthesia venous access was obtained with a 16 gauge cannula sited in the antecubital fossa. A separate intravenous cannula was sited in the contralateral hand for administration of intravenous anaesthetic agents. Patients were then oxygenated with $100 \%$ oxygen for $3 \mathrm{~min}$. Baseline venous samples were taken. Anaesthesia was induced in each patient by intravenous injections of sodium thiopentone $4 \mathrm{mg} / \mathrm{kg}$, fentanyl $1 \mu \mathrm{g} / \mathrm{kg}$ and vecuronium $0.1 \mathrm{mg} / \mathrm{kg}$. On attaining
P. Heyworth

R. Bourke

C. Moore

N. Morlet

R. Cooling

Moorfields Eye Hospital London, UK

Mr Peter Heyworth 82 Manchuria Road London SW11 6AE, UK

Tel/fax:

$+44(0) 1712281208$

Sponsored by the Moorfields Locally Organised Research Fund 
Table 1. The age, weight and volume of posterior segment infusion for the HS + Ad group and the Hs group

\begin{tabular}{ccccc}
\hline Patient group & Age (years) & Weight $(\mathrm{kg})$ & Volume infused (ml) & Quantity of adrenaline infused (mg) \\
\hline Hs + Ad & $61.3(39-90)$ & $75.3(63-103)$ & $120(40-200)$ & $0.12(0.04-0.2)$ \\
Hs & $64(5575)$ & $56.1(46.5-84)$ & $120(100-150)$ & - \\
\hline
\end{tabular}

Values are the mean (range). HS+Ad, Hartmann's solution + adrenaline; Hs, Hartmann's solution only.

muscular paralysis, as demonstrated by the loss of a train of four nerve stimulations, tracheal intubation was performed. Anaesthesia was maintained using intermittent positive pressure ventilation with a combination of $66 \%$ nitrous oxide and $33 \%$ oxygen whilst enflurane was maintained between $0.5 \%$ and $2 \%$ to keep pulse and blood pressure within normal limits. End-tidal carbon dioxide was maintained between $4.0 \%$ and $4.4 \%$.

Peripheral venous blood samples were taken at the following intervals: prior to induction of anaesthesia, 5 min following intubation, and at 5, 10, 15 and $30 \mathrm{~min}$ following the commencement of infusion. Samples were placed without delay into lithium heparin tubes containing the anti-oxidant sodium metabisulphite and immediately cooled on ice for $5 \mathrm{~min}$ before being centrifuged at 3000 r.p.m. The serum was separated with a pipette and placed in a plain tube on ice for a maximum of $2 \mathrm{~h}$ before being frozen to $-15^{\circ} \mathrm{C}$. Samples were stored for a maximum of 1 month before assay for adrenaline levels using high-performance liquid chromatography with an ESA Coulochem electrochemical detector.

Patients had pulse, blood pressure and electrocardiograms monitored at 3 min intervals from the pre-induction stage. The timings of the blood samples were recorded and the total volume of posterior segment infusion was recorded at each sampling time.

Comparisons of adrenaline levels between the two groups were made using Wilcoxon's signed rank test.

\section{Results}

Ten patients were studied. Six patients were randomised to the Hs+Ad group and four the the Hs group.

The mean age in the $\mathrm{Hs}+\mathrm{Ad}$ group was 61.3 years (range 39-90 years) with a male-to-female ratio of 2:1. Mean weight was $75.3 \mathrm{~kg}$ (range 63-103 kg). The mean age in the Hs group was 64 years (range 55-75 years) with a male-to-female ratio of 1:3. Mean weight was 56.1 $\mathrm{kg}$ (range 46.5-84 kg).

The mean volume of infusion in the Hs+Ad group was $120 \mathrm{ml}$ (range $40-200 \mathrm{ml}$ ). The mean quantity of adrenaline infused was $655.1 \mathrm{nmol}(0.12 \mathrm{mg})$, range 218.4-1091.8 nmol (0.04-0.2 mg). The mean volume of infusion in the Hs group was $120 \mathrm{ml}$ (range 100-150 ml) (Table 1).

Serum adrenaline levels at the various sampling times are shown for both groups in Table 2. The maximum coefficient of variation for adrenaline levels below $1 \mathrm{nmol} / \mathrm{l}$ using the Coulochem detector was $8 \%$. Adrenaline levels follow a non-Gaussian distribution due to the lowest sensitivity of the assay being $0.1 \mathrm{nmol} / 1$. This results in a cut-off effect with bunching of data at the $0.1 \mathrm{nmol} / 1$ level. Adrenaline levels remained within physiological limits for all samples.

Adrenaline levels for all sampling times grouped together following the commencement of infusion were higher in the Hs+Ad group (median $=0.15 \mathrm{nmol} / \mathrm{l}$ ) than the Hs group (median $=0.10 \mathrm{nmol} / \mathrm{l}$ ); however, this difference was not statistically significant.

Median adrenaline levels were higher at each individual sampling time following infusion in the Hs + Ad group; however, this difference only approached significance at the infusion $+15 \min (p=0.05)$ and infusion +30 min sampling times $(p=0.05)$.

There was no statistical difference between adrenaline levels prior to induction or following intubation and subsequent post-infusion levels in either group. There was no significant change in adrenaline level associated with changes in infusion rate throughout the procedure. Furthermore there was no detectable association between

Table 2. The serum adrenaline concentrations detected at various intervals for each patient in the two groups

\begin{tabular}{|c|c|c|c|c|c|c|c|c|}
\hline Patient no. & Pre-induction & $\begin{array}{l}\text { Intubation } \\
+5 \mathrm{~min}\end{array}$ & $\begin{array}{c}\text { Median } \\
\text { pre-infusion }\end{array}$ & $\begin{array}{l}\text { Infusion } \\
+5 \mathrm{~min}\end{array}$ & $\begin{array}{l}\text { Infusion } \\
+10 \mathrm{~min}\end{array}$ & $\begin{array}{l}\text { Infusion } \\
+15 \text { min }\end{array}$ & $\begin{array}{l}\text { Infusion } \\
+30 \text { min }\end{array}$ & $\begin{array}{l}\text { Median post- } \\
\text { infusion }\end{array}$ \\
\hline \multicolumn{9}{|l|}{ Hs + Ad group } \\
\hline 1 & 0.36 & 0.41 & 0.38 & 0.36 & 0.25 & 0.30 & 0.19 & 0.28 \\
\hline 2 & 0.10 & 0.10 & 0.10 & 0.10 & 0.10 & 0.12 & 0.25 & 0.11 \\
\hline 3 & 0.10 & 0.10 & 0.10 & 0.10 & 0.10 & 0.25 & NA & 0.10 \\
\hline 4 & 0.10 & 0.10 & 0.10 & 0.10 & 0.10 & 0.10 & 0.10 & 0.10 \\
\hline 5 & 0.29 & 0.31 & 0.30 & 0.35 & 0.15 & 0.24 & 0.14 & 0.19 \\
\hline 6 & 0.66 & 0.23 & 0.45 & 0.22 & 0.20 & 0.16 & 0.15 & 0.18 \\
\hline $\begin{array}{l}\text { Median } \\
\text { (patients 1-6) } \\
\text { Hs group }\end{array}$ & 0.20 & 0.17 & 0.17 & 0.16 & 0.13 & 0.20 & 0.15 & 0.15 \\
\hline 7 & 0.10 & 0.10 & 0.10 & 0.10 & 0.10 & 0.10 & 0.10 & 0.10 \\
\hline 8 & 0.50 & 0.13 & 0.31 & 0.12 & 0.13 & 0.10 & 0.10 & 0.11 \\
\hline 9 & 0.20 & 0.50 & 0.28 & 0.10 & 0.13 & 0.12 & 0.10 & 0.11 \\
\hline 10 & 0.15 & 0.10 & 0.12 & 0.10 & 0.10 & 0.10 & 0.10 & 0.10 \\
\hline $\begin{array}{l}\text { Median } \\
\text { (patients 7-10) }\end{array}$ & 0.18 & 0.12 & 0.14 & 0.10 & 0.12 & 0.10 & 0.10 & 0.10 \\
\hline
\end{tabular}


adrenaline infusion rate and pulse or blood pressure under these standardised anaesthetic conditions. No cardiac dysrhythmias were detected in either group.

\section{Discussion}

Adrenaline is frequently added to the indwelling posterior segment infusion during vitreoretinal procedures in order to maintain peroperative mydriasis and assist with haemostasis. Typically $0.5 \mathrm{ml}$ of a 1:1000 solution $(0.5 \mathrm{mg})$ is added to $500 \mathrm{ml}$ of Hartmann's solution for this purpose. For comparison, a typical resuscitative intravenous dose of adrenaline is $1 \mathrm{mg}$.

When infused through the posterior segment there are many potential routes by which the adrenaline can be absorbed into the systemic circulation and give rise to systemic effects. These include through the conjunctival membrane, the nasal mucous membrane and gastrointestinal tract as well as via the ciliary body, choroid and iris. Anaesthetists are justifiably cautious about the use of adrenaline in this manner, particularly in patients with poorly controlled hypertension, those with cardiac dysrhythmias or those receiving halothane anaesthesia. ${ }^{1}$

A review of the literature yields no information regarding adrenaline absorption following this specific route of administration; however, there are several reports of significant absorption and systemic effects when adrenaline is administered in the ophthalmic setting. ${ }^{2-5}$

A dose of $0.5 \mathrm{mg}$ adrenaline administered subcutaneously can result in a 6-fold increase in serum adrenaline levels after 5 min with associated rises in blood pressure and pulse rate. ${ }^{6}$ Similarly when $0.05 \mathrm{mg}$ (10 $\mathrm{ml}$ of 1:200 000 ) of adrenaline is added to a retrobulbar block there is a 3 -fold increase in serum adrenaline levels, which is similar to the rise associated with psychological stress. ${ }^{7}$ Even as little as $0.075 \mathrm{mg}$ when injected into highly vascularised tissues can lead to an 8 -fold rise in serum levels after 2 min. $^{8}$

To achieve ocular penetration ophthalmic drops contain high concentrations of adrenaline. One drop of adrenaline $2 \%$ contains $1 \mathrm{mg}$ of adrenaline base. With the delivery of as little as one drop of $2 \%$ adrenaline $25 \%$ of patients develop a significantly elevated systolic blood pressure and a significant increase in the number of ventricular ectopic beats. ${ }^{4}$ These effects would appear to be idiosyncratic. Other workers have also reported dangerous elevations of pulse and blood pressure in healthy young adults. ${ }^{2,3}$ Drop application under general anaesthesia may result in enhanced systemic absorption due to the absence of voluntary tear wiping and a blink reflex. ${ }^{9}$ Despite these significant clinical effects one study failed to demonstrate significant elevations of serum adrenaline levels following topical ocular application of adrenaline $1 \mathrm{mg} .{ }^{6}$ Using topical radiolabelled adrenaline in a rabbit model it has been shown that $55 \%$ is absorbed into the circulation over a period of several hours. The major pathway appears to be through the gastrointestinal tract. ${ }^{5}$ What is not known, however, is whether this absorption leads to recordable serum levels that may give rise to unwanted cardiac effects.

In this study we were unable to demonstrate a clinically significant rise in serum adrenaline levels following posterior segment infusion despite the many potential routes for absorption. Although the assay used in this study did not distinguish between endogenous adrenaline secretion and exogenous absorption there did not appear to be a significant difference between preoperative adrenaline levels and post-infusion levels in either group. The lack of a pre-operative stress-associated rise could be accounted for by the anxiolytic effect of the pre-medication.

The existing literature suggests that the total dose delivered in this surgical setting could be enough to produce recordable rises in adrenaline levels and result in significant systemic effects. We failed to detect a significant rise in adrenaline levels amongst those receiving an adrenaline infusion. The levels were, however, higher in the group receiving infused adrenaline and approached significance at 15 and $30 \mathrm{~min}$ after infusion. This suggests that there may have been some absorption but at a very slow rate. The reasons for a lack of significant rise in adrenaline levels could be twofold. Firstly the total load and its rate of delivery were low: the dilution of the infused adrenaline was $0.5 \mathrm{mg} / 500 \mathrm{ml}$ and the maximum rate of delivery was $16 \mathrm{ml} / \mathrm{min}(0.016 \mathrm{mg} / \mathrm{min})$. This is very low compared with the instant delivery of a topical drop containing $1 \mathrm{mg}$ adrenaline base. Secondly adrenaline is metabolised very rapidly in the serum and liver by catecholamine methyltransferase, resulting in a half-life of less than $5 \mathrm{~min}$.

We did not detect any significant changes in pulse rate or blood pressure that could be attributed to the adrenaline infusion and there were no detected incidences of cardiac dysrhythmia. Due to the standardisation of the anaesthetic by parameters that included pulse and blood pressure little relevance can be applied to these particular findings.

We conclude from this study that there is no significant absorption of adrenaline when infused through the posterior segment at the described rates and concentrations. Adverse reactions to adrenaline may, however, be idiosyncratic. ${ }^{4}$ Continued caution must be exercised when using this drug in posterior segment infusion during vitreoretinal surgery.

\section{References}

1. Smith RB, Douglas J, Petruscak J, Breslin P. Safety of intraocular adrenaline with halothane anaesthesia. $\mathrm{Br}$ J Anaesth 1972;44:1314-6.

2. Ballin N, Becker B, Goldman ML. Systemic effects of epinephrine applied topically to the eye. Invest Ophthalmol 1966;5:125-9.

3. Lansche RK. Systemic reactions to topical epinephrine and phenylephrine. Am J Ophthalmol 1966;62:95-8. 
4. Kerr CR, Hass I, Drance SM. Cardiovascular side effects of epinephrine and dipivalylepinephrine applied topically to the eye in patients with glaucoma. Br J Ophthalmol 1982;66: 109-14.

5. Anderson JA. Systemic absorption of topically applied epinephrine and dipivefrine. Arch Ophthalmol 1980;98:350-1. 6. Dahlof C, Mellstrand T, Svendmyr N. Systemic absorption of adrenaline after aerosol, eye-drop and subcutaneous administration to healthy volunteers. Allergy 1987;42:215-21.
7. Donlon JV, Moss J. Plasma catecholamine levels during local anaesthesia for cataract operations. Anaesthesiology 1979;51:471-3.

8. Low JM, Harvey JT, Cooper GM, Prenderville WJ. Plasma concentrations of catecholamines following adrenaline infiltration during gynaecological surgery. $\mathrm{Br} \mathrm{J}$ Anaesth 1984;56:849-52.

9. Webster NR, Hinton W. Topical adrenaline [letter]. Anaesthesia 1986;41:435. 